\title{
Evaluación de la capacidad desulfurante de la cascarilla de Oryza sativa transformada como material poroso activado con potencial uso en el tratamiento de biogás.
}

Evaluation of desulfuring capacity of the Oryza sativa shell transformed as a activated porous material with potential use in the treatment of biogas.

José Luis Manrique Murillo, Oscar Christian Medina Carpio, Pamela Lisbeth Manrique Pino, Ever Pavel Macedo Benavente, Danny Pamela Tupayachy Quispe.

Universidad Católica de Santa María. Arequipa Perú.

\section{INFORMACIÓN}

\section{Historia del Artículo}

Recepción: 12/12/2019

Revisión: 10/02/2020

Aceptación: 20/05/2020

\section{Palabras Clave}

Adsorción, Biogás, Carbón Activado,

Filtros y cascarilla de arroz.

\section{Key Words}

Adsorption, Biogas, Activated Carbon, Filters and rice husk.

\section{DOI}

https://doi.org/10.35286/veritas. v21i2.281

\begin{abstract}
RESUMEN
La cascarilla de Oryza sativa suele ser desechada e incinerada sin control por lo cual en la presente investigación se le dio un uso alternativo mediante su transformación como material adsorbente codificado como HRKOH, ACARROZ y ACFeO. (CAC para carbón activado comercial), la activación de la cascarilla se llevó a cabo de manera química utilizando $\mathrm{KOH}$ (Potasa Caustica "Clint" en escamas) al 40\% esto para lisar estructuras lignocelulósicas; posteriormente se adicionó químicamente un óxido metálico. El proceso se optimizó mediante un diseño factorial multinivel, el rendimiento máximo lo registró el material HRKOH y fue de $94.45 \%$, no obstante debido a su escasa porosidad y consiguientemente baja retención de contaminantes no se le consideró como un material competente. Las pruebas en campo respecto a la adsorción de ácido sulfhídrico mostraron similares comportamientos entre $A C a r r o z, A C F e O$ y $C A C$, es así que se optó por contrastar al material ACARROZ vs CAC mediante isotermas obteniéndose ajustes similares para ambos materiales; el ACarroz se ajustó mejor a la isoterma de Langmuir mostrando un R2 de 0.9937 (R2 en Freundlich = 0.986), comportamiento similar mostró el carbón comercial al ajustarse con un coeficiente de determinación de 0.9965 para Langmuir y 0.957 para Freundlich.
\end{abstract}

\section{INTRODUCCIÓN}

El Ácido sulfhídrico (H2S) es un gas incoloro y tóxico más pesado que el aire que suele presentarse como un agregado indeseable en la producción de biogás terminando por dañar estructuras, si bien los métodos actualmente desarrollados son eficaces en la depuración de H2S no ofrecen alternativas ecológicas, de bajo costo y sobre todo de reaprovechamiento de los recursos naturales de la región. (Elaboración propia)

Correspondencia:

Oscar Christian Medina Carpio

omedinac@ucsm.edu.pe
La presencia de $\mathrm{H} 2 \mathrm{~S}$ en el biogás supone un riesgo a la salud y al medio ambiente ya que el biogás no representa un problema en sí mismo, de hecho se presenta como una alternativa ecológica al gas común derivado del petróleo y su producción no debiese representar ningún riesgo ambiental pero la presencia de $\mathrm{H} 2 \mathrm{~S}$ en él, superior a $10 \mathrm{ppm}$ se traduce en serios problemas para el operario tanto en el manejo como en el procesamiento del biogás por su toxicidad, alto poder corrosivo, disminución del poder calorífico del gas, generación de olores desagradables y tóxicos dentro de muchas indeseables características. (KGaA)

En la actualidad existen métodos como lavados químicos, adsorción en lechos, tratamientos biológicos, entre otros; que se enfocan en la desulfuración de efluentes gaseosos, pero 
no llegan a satisfacer las necesidades debido a que, si bien consiguen desulfurar eficazmente el biogás no aprovechan los recursos de la región Arequipa. (Elaboración propia)

Es por ello, que la presente investigación busca brindarle un uso alterno a un subproducto de la industria arrocera como lo es la cascarilla del Oryza sativay transformarla en un material poroso que remueva el ácido sulfhídrico presente en el Biogás.

Actualmente las investigaciones realizadas respecto al tema no toman en cuenta las interacciones de diversos factores y niveles que existen para cada prueba, es por ello por lo que este proyecto utilizó un diseño factorial que permitió reducir el número de ensayos.

Por lo tanto, esta investigación propone un procedimiento eficaz para la producción de un material de calidad específica a partir de un sub producto agrícola como lo es la Cascarilla de Oryza sativa, así como el diseño de filtros piloto que contendrán matrices de distintas calidades que captarán prioritariamente Sulfuro de Hidrógeno de efluentes primordialmente gaseosos.

El presente trabajo de investigación busca generar indicadores que generen la posibilidad de desarrollar estudios posteriores de factibilidad para la implantación de una planta biotecnológica productora de diversas calidades de Carbón.

\section{MATERIALES Y MÉTODOS}

\section{Producción de los materiales adsorbentes}

Se produjeron tres materiales adsorbentes los cuales fueron denominados bajo una codificación determinada, HRKOH para un material basado en el lavado de la cascarilla de arroz con potasa cáustica, ACarroz para denominar a una calidad de carbón activado proveniente de calcinar el material previamente mencionado y $\mathrm{ACFeO}$ para denominar un carbón activado hecho a base de cascarilla de arroz, pero impregnado en moléculas de un óxido metálico específico, para el caso de la presente investigación, se optó por óxido de fierro.

\section{Obtención del primer material}

Se realizó empleando cascarilla de Oryza sativa como materia prima, para la activación se utilizó Potasa Caustica $(\mathrm{KOH})$ al $40 \%$ como agente activante, se dejó en reposo entre 4 y 8 horas. Luego de ello se escurrió el exceso de líquido con gasa y se desecó en mufla a $70^{\circ} \mathrm{C}$ por 24 horas.

\section{Obtención del segundo material adsorbente}

Se obtuvo mediante calcinación, La carbonización de un material con elevado porcentaje de carbono fijo genera varios núcleos aromáticos y dependiendo de la temperatura y tiempos de calcinación se obtendrán diferentes porosidades; dicha calcinación se llevó a cabo según la metodología propuesta por Manrique Pino a $600{ }^{\circ} \mathrm{C}$ durante treinta minutos.

\section{Obtención del tercer material Absorbente}

Es necesario resaltar que el carbón activado impregnado en óxido de fierro tiene cierta eficacia reportada en la captación de arsénico, plomo, mercurio entre otros (Reed \& Jiang, 2000). Es por lo que una fracción del segundo material adsorbente (CAarroz) se impregnó con $\mathrm{FeO}$ generando así un tercer material.

\section{Pruebas en Campo}

La interacción de los materiales con el biogás se realizó en la planta del Fundo "La Católica" (Majes - El Pedregal) se utilizó el Analizador de Gases (BIOGAS5000) provisto por la Universidad Católica de Santa María con la ayuda del cual se obtuvieron lecturas de Metano (CH4), Dióxido de carbono (CO2), Oxígeno (O2), y Sulfuro de Hidrógeno (H2S) (éste último en ppm). A continuación, en la tabla 1 se muestran los factores y diseño experimental.

Tabla 1: Factores y Niveles del diseño experimental.

\begin{tabular}{ccccc}
\hline FACTORES & -1 & -0.333 & 0.333 & 1 \\
\hline A: Tipo de Adsorbente & HRKOH & ACARROZ & ACFeO & CAC \\
B: Limaduras de Hierro & SIN & & & CON \\
\hline
\end{tabular}

\section{Isotermas de Adsorción}

El comportamiento del Carbón Activado obtenido de cascarilla de arroz se comparó con el de un Carbón Activado Comercial ya posicionado en el mercado (Carbón Activado Pulverizado - "ChemiLab") esto se determinó mediante isotermas, dichas isotermas se obtuvieron de un Test de Adsorción de Azul de Metileno provisto por la Universidad Católica Santa María y el Hospital Carlos Alberto Seguín Escobedo.

Se trabajó con Azul de Metileno Comercial Líquido cuya concentración de fábrica es de $1.1 \mathrm{~g} / \mathrm{L}$; se hizo un barrido en el espectrofotómetro BOECO S200 UV/VIS de la solución stock para determinar la longitud de onda a la cual se procederían a leer el resto de las muestras.

Las pruebas en azul de metileno son comunes en la industria para caracterizar materiales porosos como polvos minerales o carbones activados, es por ello que se llevó a cabo una metodología autogenerada que significó realizar diluciones hasta obtener un rango de concentraciones que permitirían tener un coeficiente de determinación (R2), una línea de tendencia y una ecuación de la recta para posteriormente intrapolar datos. 


\section{RESULTADOS}

\section{Evaluación del rendimiento en cada etapa del proceso productivo de cada material fabricado.}

Para evaluar los rendimientos en cada etapa del proceso se pesaron los gramajes de cada material adsorbente. Con el fin de eliminar cada vez más componentes de la materia prima es que se llevó a cabo una metodología en serie donde se registraba cada vez que se perdía peso, el pesaje se realizó por triplicado, posteriormente se llevó a cabo un análisis de varianza para determinar la significancia de los distintos pesos.

A continuación, en la tabla 2 se muestran los datos procesados en términos de porcentaje.

Tabla 2: Rendimientos de los distintos materiales adsorbentes.

\begin{tabular}{ccccc}
\hline Repetición & Cascarilla & HRKOH & ACARROZ & ACFeO \\
\hline 1 & $100 \%$ & $93.53 \%$ & $46.33 \%$ & $48.95 \%$ \\
2 & $100 \%$ & $91.22 \%$ & $46.18 \%$ & $48.65 \%$ \\
3 & $100 \%$ & $95.61 \%$ & $46.65 \%$ & $48.59 \%$ \\
\hline
\end{tabular}

Se realizó una prueba ANOVA para determinar la verdadera significancia entre los distintos datos respecto al rendimiento, se observó que, el HRKOH tiene un rendimiento mayor, esto se debe a que el material no ha pasado por un proceso de calcinación, por la cual no ha logrado deshacerse el material volátil ni se han generado la misma cantidad y calidad de poros que sí han generado los otros dos materiales (Tabla 3).

Tabla 3: Análisis de la Varianza para el rendimiento de los distintos materiales

\begin{tabular}{cccccc}
\hline Fuente & $\begin{array}{c}\text { Suma de } \\
\text { Cuadrados }\end{array}$ & GL & $\begin{array}{c}\text { Cuadrado } \\
\text { Medio }\end{array}$ & Razón F & Valor P \\
\hline $\begin{array}{c}\text { Entre grupos } \\
\text { Intra }\end{array}$ & 7325.1 & 3 & 2441.7 & 1986.23 & 0.00 \\
grupos & 9.8345 & 8 & 1.22932 & & \\
$\begin{array}{c}\text { Total } \\
\text { (correlación) }\end{array}$ & 7334.9 & 11 & & & \\
\hline
\end{tabular}

Pruebas en campo de la capacidad de adsorción de H2S de los distintos filtros y medición de su interacción con otros componentes gaseosos presentes en el Biogás.

Las pruebas en campo se llevaron a cabo siguiendo una matriz basada en el diseño de bloques completamente aleatorizados; Para dichas pruebas se utilizó un Analizador portátil por infrarrojos para biogás, BIOGAS 5000 (Tabla 4).
Tabla 4: Matriz ordenada de resultados obtenidos en las lecturas en campo.

\begin{tabular}{cccccccc}
\hline Bloque & $\begin{array}{c}\text { Tipo de } \\
\text { adsorbente }\end{array}$ & Limadura & $\begin{array}{c}\mathrm{H} 2 \mathrm{~S} \\
(\mathrm{ppm})\end{array}$ & $\begin{array}{c}\mathrm{CO} 2 \\
(\%)\end{array}$ & $\begin{array}{c}\mathrm{CH} 4 \\
(\%)\end{array}$ & O2 (\%) Bal (\%) \\
\hline 1 & -1 & -1 & 574 & 37.5 & 56.2 & 1 & 5.3 \\
1 & -0.333 & -1 & 59 & 35.6 & 54.3 & 1.5 & 8.6 \\
1 & 0.333 & -1 & 12 & 35 & 55.5 & 1.4 & 8.2 \\
1 & 1 & -1 & 17 & 14.8 & 25.7 & 12.9 & 46.5 \\
1 & -1 & 1 & 547 & 37.2 & 55.9 & 1.2 & 15 \\
1 & -0.333 & 1 & 75 & 27.8 & 42.4 & 4.8 & 25 \\
1 & 0.333 & 1 & 53 & 30 & 55.6 & 1.2 & 16.9 \\
1 & 1 & 1 & 44 & 11.2 & 27.3 & 13.8 & 48.5 \\
2 & -1 & -1 & 602 & 35 & 54.5 & 1.2 & 6.2 \\
2 & -0.333 & -1 & 6 & 27.3 & 49.8 & 3.6 & 19.3 \\
2 & 0.333 & -1 & 6 & 35.6 & 56.8 & 1.1 & 6.5 \\
2 & 1 & -1 & 15 & 13.9 & 27.5 & 11.4 & 47.1 \\
2 & -1 & 1 & 705 & 39 & 53.8 & 0.8 & 16.2 \\
2 & -0.333 & 1 & 16 & 28.9 & 53.5 & 1.4 & 21 \\
2 & 0.333 & 1 & 123 & 33.7 & 44.2 & 0.7 & 13.8 \\
2 & 1 & 1 & 49 & 14.9 & 22.5 & 12 & 51.6 \\
\hline
\end{tabular}

Las pruebas en campo fueron satisfactorias dado que el ACarroz, el ACFeO y el CAC mostraron un excelente comportamiento en la captación de $\mathrm{H} 2 \mathrm{~S}$ al mostrar promedios de $39 \%, 48.5 \%$ y $31.25 \%$ de $\mathrm{H} 2 \mathrm{~S}$ libre, respectivamente; a su vez sus interacciones con el metano son mínimas excepto por el CAC ya que es el material que más metano captura deja solo un $25.75 \%$ libre.

Pruebas de adsorción de azul de metileno e Isotermas de Adsorción para ACARROZ vs CAC.

Se trabajó con diluciones de azul de metileno con concentraciones de 300, 500, 700, 900 y $1100 \mathrm{mg} / \mathrm{L}$ (Azul de metileno/Agua) las cuáles interactuaron con 1 gr de material adsorbente, se agregaron alícuotas cada 15 minutos y se obtuvieron absorbancias a partir de las cuales se logró modelar isotermas de Freundlich y Langmuir.

Herbert Freundlich describió un ajuste para entender los procesos de adsorción, dicho ajuste responde a una adsorción en bicapa, es decir que la adsorción ocurre en una capa inicial y posteriormente se produce una condensación (Tabla 5) (Figura 1).

Tabla 5: Tabla de Valores para Modelo F del ACARROZ

\begin{tabular}{cccc}
\hline Co & Ce $[\mathrm{AM}] \mathrm{mg} / \mathrm{L}$ & Qe $(\mathrm{mg} / \mathrm{g})$ & Modelo $\mathrm{F}$ \\
\hline 300 & 0.217 & 29.978 & 31.006 \\
500 & 4.459 & 49.554 & 47.332 \\
700 & 39.915 & 66.409 & 63.281 \\
900 & 164.164 & 73.584 & 78.188 \\
110 & 262.626 & 83.737 & 83.473 \\
\hline
\end{tabular}




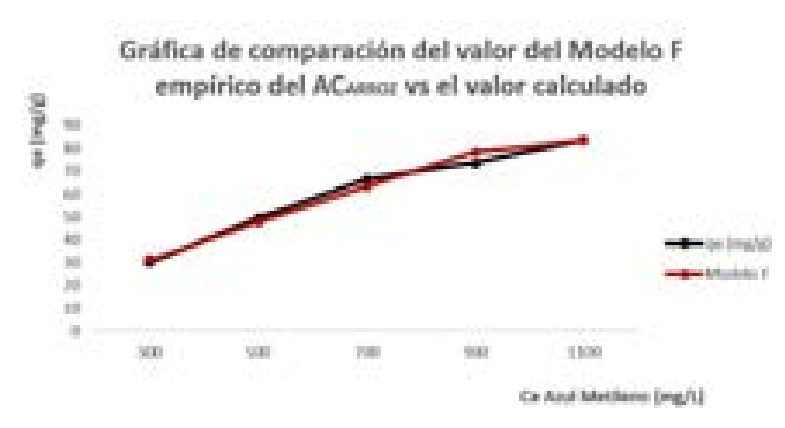

Fig. 1: Comparacion del valor del Modelo F empírico de AC arroz vs el valor calculado

Irving Langmuir postulo una isoterma que mereció un premio nobel de química, dicho modelo asume un número inmenso, casi infinito de probables sitios activos sobre los cuales se unirá el adsorbato, por lo que se puede hablar de un tipo de adsorción en monocapa ya que todos estos sitios se encontrarían dispersos de manera homogénea en la superficie (Tabla 6) (Figura 2).

Tabla 6: Tabla de Valores para Modelo L para ACARROZ

\begin{tabular}{ccccc}
\hline $\mathrm{Co}$ & $\mathrm{Ce}[\mathrm{AM}] \mathrm{mg} / \mathrm{L}$ & $\mathrm{Qe}(\mathrm{mg} / \mathrm{g})$ & $\mathrm{Ce} / \mathrm{qe}$ & Modelo L \\
\hline 300 & 0.217 & 29.978 & 0.007 & 3.017 \\
500 & 4.459 & 49.554 & 0.090 & 36.224 \\
700 & 39.915 & 66.409 & 0.541 & 71.301 \\
900 & 164.164 & 73.584 & 2.231 & 79.865 \\
110 & 262.626 & 83.737 & 3.316 & 80.885 \\
\hline
\end{tabular}

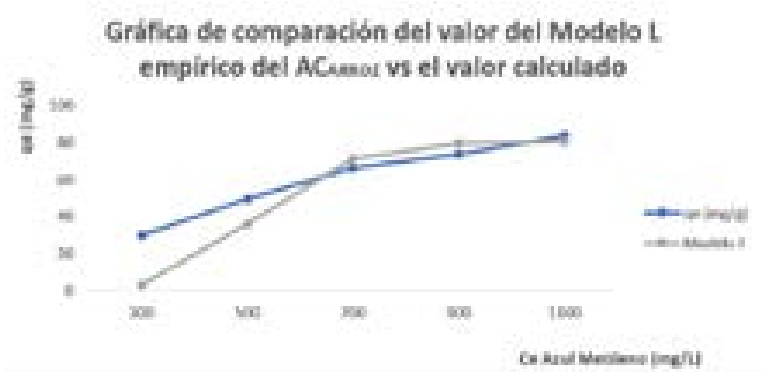

Fig. 2: Comparacion del valor del Modelo L empírico de AC arroz vs el valor calculado

\section{DISCUSIÓN}

Debido al alto poder corrosivo del Ácido Sulfhídrico es que el biogás no puede ser utilizado ni en calderas, ni en motores, ni en las celdas de combustible, etc, es por ello debe ser removido del biogás. (1)

La opciones analizadas para absorber el Ácido Sulfhídrico tales como el carbón activado comercial y el carbón activado producido a partir de cascarilla de arroz parecen no diferir en su adsorción, lo mismo que el ACFeO; en 2007 Qiao Li Zhang y su equipo de investigación de la Universidad de Tongji en Shangai - China diseñaron un método para preparar un carbón activado impregnado de moléculas de Óxido de Fierro y evaluaron su capacidad adsortiva de Arsénico y si bien su material se ajustaba perfectamente a la isoterma de Langmuir y mostraba alentadores datos respecto a la adsorción, la presencia de un oxido metálico parecía no afectar signicativamente al área superficial ni a la estructura porosa del carbón activado. (2) A su vez, Haifeng Zhuan, también en china, propuso la preparación de un carbón activado soportado en Óxido de Fierro y Manganeso como catalizadores (Haifeng Zhuang, 2014); ello corrobora la relevancia de modificar al carbón activado comercial mediante la adición de un óxido metálico.

Las limaduras de hierro utilizadas fueron recolectadas de un centro de procesamiento de metales de la región Arequipa, a pesar de haber sido lavadas y separadas del polvo y de la tierra con un imán, no sufrieron una mayor transformación y fueron utilizadas en su estado puro dentro de los filtros y es por ello que pudieron no haber mostrado interacción alguna en la adsorción ya que Vázquez Valencia et all al diseñar todo un sistema de purificación de Biogás lavaron las limaduras con $\mathrm{NaOH}$ para que el óxido adquiera la configuración $\mathrm{Fe} 2 \mathrm{O} 3$ que interactuará rápidamente con el H2S. (L. Vázquez Valencia, 2015)

Para el HRKOH se buscó eliminar las impurezas y lisar ciertas moléculas como la celulosa y sílice que la constituyen (3); el rendimiento de este primer material fue de 95.45 $\%$, es decir que el material lisado y arrastrado junto con el çhar se aproximó al $5.55 \%$. La bibliografía propone una composición química de la cascarilla en la cual se indica que la misma está conformada por un $39.1 \%$ de carbono, $5.2 \%$ de hidrógeno, $0.6 \%$ de nitrógeno, $\%$ de oxígeno, un $0.1 \%$ de Azufre y el $17.8 \%$ restante de cenizas. (Cortez, 2010)

El HRKOH es el material que reporta el más bajo desempeño en contraste con los otros materiales producidos en este trabajo respecto a la captación de $\mathrm{H} 2 \mathrm{~S}$ al dejar un aproximado de $607 \mathrm{ppm}$ libres de lecturas iniciales de aproximadamente $1000 \mathrm{ppm}$. Su interacción con el metano, a pesar de ser mínima, perfila al HRKOH como una opción a descartar debido a que se busca una casi nula adsorción de $\mathrm{CH} 4$ porque de otro modo el biogás perdería toda capacidad de ser combustible.

Para entender bien el estudio de la interacción de los distintos materiales con el oxígeno presente por defecto en el biogás es necesario entender que el biogás suele tener una composición promedio que consta de metano (CH4) 50-70 $\%$, dióxido de carbono (CO2) 30-50\%, nitrógeno (N) 0.5-3 $\%$, ácido sulfhídrico (H2S) 0.1- $2 \%$, hidrogeno (H) 1-2\% (6). El oxígeno es un elemento poco presente en el biogás (2-4\%) y es que al trabajar con concentraciones mayores al $11 \%$ de oxigeno se aumenta el riesgo de explosividad del biogás, esto según diversos manuales de seguridad en plantas de producción y procesamiento de gás y biogás. (7) (BRADFER, 2002).

\section{CONCLUSIONES}

El ACARROZ se presenta como la mejor alternativa para reemplazar a un carbón activado comercial ya que no muestra comportamientos inferiores respecto al $\mathrm{ACFeO}$ y este último supone un encarecimiento del producto ya que se requiere de ciertos insumos adicionales para su producción.

Tanto el Carbón Comercial como el producido en base a cascarilla de arroz se ajustan mejor a la isoterma de Langmuir, ambos tienen un factor de determinación de 0.957 
y 0.986 respectivamente bajo el modelo de Freundlich, son en sí valores más que aceptable, sin embargo, sus valores de R2 para Langmuir son de 0.9965 para el carbón comercial y 0.9937 para el carbón hecho de cascarilla; todo ello permite afirmar que ambos carbones adsorben en monocapa, es decir que tienen un número infinito de sitios activos en su superficie.

\section{REFERENCIAS BIBLIOGRÁFICAS}

1. FAO GdC, GEF. Manual de Biogás. Chile: Remoción de Barreras para la Electrificación Rural con Energía Renovables. Ministerio de Energía - Gobierno de Chile;. Available from: http://www.fao.org/docrep/019/ as400s/as400s.pdf.

2. Zhang QL, Lin Y, Chen X, Gao NY. A method for preparing ferric activated carbon composites adsorbents to remove arsenic from drinking water. Journal of hazardous materials. 2007;148(3):671-678.

3. Prada-Matiz A, Castillo CEC. La descomposición térmica de la cascarilla de arroz: una alternativa de aprovechamiento integral. Orinoquia. 2010;14(2):155170.

4. Degiovanni B VM, Martínez R CP, Francisco MO. Producción eco-eficiente del arroz en América Latina. Ciat.

5. Valverde A, Sarria B, Monteagudo JP. Análisis comparativo de las características fisicoquímicas de la cascarilla de arroz. Scientia et technica. 2007;1(37).

6. Arvizu J, Huacuz JM. Biogás de rellenos sanitarios para producción de electricidad. Boletín IIE. 2003;27(4):118-123.

7. Manuel Maciejczyk GGAAdB. Seguridad en Plantas de Biogás: Peligros y Medidas de Seguridad;. Available from: https://www.4echile.cl/4echile/wp-content/ uploads/2017/11/2_Hazards-and-Safety-Measures Maciejczyk_ES.pdf. 